\title{
Caracterização clínica, diagnóstico histopatológico e distribuição geográfica das neoplasias mamárias em cadelas de Salvador, Bahia ${ }^{1}$
}

\author{
Julia Morena de Miranda Leão Toríbio², Alessandra Estrela Lima', Emanoel Ferreira Martins Filho,, \\ Lorena Gabriela Rocha Ribeiro ${ }^{5}$, Mário Jorge Melhor Heine D'Assis' ${ }^{2}$, Raquel Graça Teixeira², \\ Karine Araújo Damasceno ${ }^{6}$, Geovanni Dantas Cassali ${ }^{7}$, João Moreira da Costa Neto ${ }^{8}$
}

\section{RESUMO}

Os tumores espontâneos da glândula mamária representam, nas cadelas, a neoplasia mais observada na clínica de pequenos animais, justificando o grande interesse dos pesquisadores no estudo dessa lesão. Este trabalho teve por objetivo determinar a frequência dos tumores mamários em cadelas atendidas no HOSPMEV/UFBA, no município de Salvador, no período de janeiro de 2006 a abril de 2008, bem como realizar a caracterização clínica, a classificação histopatológica e o georreferenciamento dessa patologia. Os resultados revelaram alta prevalência de neoplasias mamárias em cadelas, em sua maioria tumores, em estádios mais avançados, sendo os carcinomas em tumores mistos o tipo histológico predominante. Observou-se ainda a correlação das características socioeconômicas da população estudada com fatores sabidamente prognósticos para neoplasias mamárias em cadelas. Esses resultados refletem a procura tardia da assistência Médica Veterinária. Por isso, é urgente educar a população quanto à prevenção e deteç̧ão da neoplasia mamária nas cadelas, buscando o diagnóstico precoce e a terapêutica adequada.

Palavras-chave: tumor de mama, estadiamento clínico, fatores socioeconômicos.

\section{ABSTRACT \\ Clinical characterization, histopathologic diagnosis and geoprocessing of mammary tumours in bitches from the city of Salvador, Bahia State}

Spontaneous tumors of the mammary gland in female dogs, represent the most frequent neoplasms observed in the small animal clinic, which explains the high interest of researchers in the study of this lesion. This study aimed to determine the frequency of mammary tumors in female dogs treated at HOSPMEV/UFBA, in Salvador, from January 2006 to April 2008, as well as to carry out the clinical characterization, histopathologic classification and the georeferencing of this pathology. The results showed a high prevalence of mammary tumors in female dogs, with most of the tumors in more advanced stages and the carcinomas in mixed tumors the predominant histologic type. There was

\footnotetext{
Recebido para publicação em 23/02/2012 e aprovado em 21/06/2012

Este trabalho é parte da dissertação de mestrado da primeira autora

${ }^{2}$ Médico(a) Veterinário(a), Mestre. Departamento de Patologia e Clínicas, Universidade Federal da Bahia, Avenida Adhemar de Barros, 500, Ondina, 40170-110, Salvador, Bahia, Brasil.jutoribio@yahoo.com.br, ariojorgeh@uol.com.br, raquelteixeira10@gmail.com

${ }^{3}$ Médica Veterinária, Doutora. Departamento de Patologia e Clínicas, Universidade Federal da Bahia, Avenida Adhemar de Barros, 500, Ondina, 40170-110, Salvador, Bahia, Brasil. alestrela@gmail.com

${ }^{4}$ Médico Veterinário, Mestre. Doutorando da Faculdade de Ciências Agrárias e Veterinárias, Universidade Estadual de São Paulo, Via de Acesso Professor Paulo Donato Castellane, s/n, 14884-900, Jaboticabal, São Paulo, Brasil. emanoelfilho@ufba.br

${ }^{5}$ Médica Veterinária, Mestre. Doutoranda do Departamento do Programa de Pós Graduação em Ciência Animal, Universidade Federal de Minas Gerais, Avenida Presidente Antônio Carlos, 6627, 31270-901, Belo Horizonte, Minas Gerais, Brasil. lorenagrr@gamil.com

${ }^{6}$ Médica Veterinária, Mestre. Doutoranda do do Programa de Pós Graduação em Patologia, Universidade Federal de Minas Gerais, Avenida Professor Alfredo Balena, 190, 30130100, Belo Horizonte, Minas Gerais, Brasil. kkyvet@gmail.com

Médico Veterinário, Doutor. Departamento de Patologia Geral, Universidade Federal de Minas Gerais, Avenida Presidente Antônio Carlos, 6627, 31270-901, Belo Horizonte, Minas Gerais, Brasil. geovanni.cassali@gmail.com

${ }^{8}$ Médico Veterinário, Doutor. Departamento de Patologia e Clínicas, Universidade Federal da Bahia, Avenida Adhemar de Barros, 500, Ondina, 40170-110, Salvador, Bahia, Brasil. jmcn@ufba.br (autor para correspondência).
} 
also a.correlation between socioeconomic characteristics of the study population with known prognostic factors for mammary neoplasms in female dogs. These results reflect the delay in providing veterinary medical care. Thus, it's important to educate the population regarding the prevention and detection of mammary cancer in female dogs looking for an early diagnosis and appropriate therapy.

Key words: mammary tumor, clinical staging, socioeconomic factors.

\section{INTRODUÇÃO}

O câncer de mama em mulheres é considerado de extrema importância para saúde pública, motivando estudos sobre prevenção e diagnóstico precoce e buscando reduzir a morbidade e mortalidade relacionadas com essa neoplasia (Humphrey et al., 2002). Também em Medicina Veterinária, tem despertado grande interesse, principalmente pela elevada prevalência de tumores malignos em cadelas, em grande parte reflexo do diagnóstico tardio, que compromete o tratamento e reduz a taxa de sobrevida dos animais (Silva et al., 2004; Cavalcanti \& Cassali, 2006; Andrade et al., 2010; Estrela-Lima, 2010).

As neoplasias mamárias em cadelas e em mulheres apresentam similaridades epidemiológicas, clínicas, biológicas e genéticas, o que possibilita a utilização da cadela como modelo comparativo experimental (Schneider et al., 1969; Peleteiro, 1994; Cassali, 2000; Misdorp, 2002; Rivera \& Von Euler, 2011).

A etiologia do câncer de mama é multifatorial, envolvendo a participação de componentes genéticos, hormonais, nutricionais e ambientais (Henderson \& Feigelson, 2000; Silva et al., 2004; Uva et al., 2009; Andrade et al., 2010). As alterações no estilo de vida da sociedade moderna contribuem com uma maior exposição da população humana a alguns fatores potencialmente carcinogênicos, havendo interferência também nos hábitos alimentares dos animais, os quais passam a estar sob riscos semelhantes (Silva et al., 2004; Dagli, 2008).

A avaliação clínica das cadelas com tumores de mama é de fundamental importância para determinar a fase de evolução da doença, fornecendo melhor entendimento sobre o comportamento biológico da neoplasia e proporcionando informações de significado diagnóstico, prognóstico e terapêutico (Allred et al., 1998). Os tumores maiores do que cinco centímetros, de rápido crescimento, aderentes, com grandes áreas de ulceração e com metástase para linfonodos estão associados ao pior prognóstico (Cavalcanti \& Cassali, 2006; Ferreira et al., 2009; Estrela-Lima, 2010).

Todavia, independentemente do tamanho tumoral, é imprescindível a realização do exame anátomo-histopatológico de todos os nódulos. Dentre os tumores mamários malignos, os mais frequentes são os carcinomas (Brodey et al., 1983; Peleteiro, 1994); entretanto, há uma grande variação na frequência dos tumores mamários de caráter benigno e maligno e os subtipos neoplásicos, em consequência da utilização de diferentes métodos de classificação histopatológica e da ausência de critérios homogêneos na diferenciação dos tipos tumorais (Cassali, 2000).

Este trabalho teve por objetivo determinar a prevalência dos tumores mamários em cadelas no município de Salvador, atendidas no HOSPMEV/UFBA, entre janeiro de 2006 e abril de 2008. Além disso, realizar a caracterização clínica e histopatológica dessa neoplasia e o georreferenciamento das cadelas acometidas, verificando a existência ou não de correlação entre as condições socioeconômicas do proprietário e o estadiamento clínico do tumor no momento da consulta.

\section{MATERIAL E MÉTODOS}

\section{Seleção e avaliação dos animais}

A pesquisa foi realizada no Hospital de Medicina Veterinária Professor Renato Medeiros Neto, pertencente à Universidade Federal da Bahia (UFBA), envolvendo os setores de Clínica e Cirurgia de Pequenos Animais, Laboratório de Anatomia Patológica (LABAP) e Laboratório de Monitoramento de Doenças pelo Sistema de Informação Geográfica (LAMDOSIG).

Inicialmente, todos os animais com lesão mamária foram submetidos ao exame clínico, que consistiu na avaliação da condição clínica geral do animal, coleta de informações sobre o histórico clínico e ciclo reprodutivo (cio regular, número de partos, castração, utilização de tratamento hormonal, abortos, período de desenvolvimento das lesões, histórico de pseudociese e lesões prévias). Essas informações foram armazenadas, em ficha oncológica específica, segundo o protocolo modificado de Ferreira et al. (2003).

No exame físico, foram feitas a avaliação do estado corporal e do porte dos animais, a identificação dos tumores mamários, quanto ao número, tamanho e localização, e a avaliação macroscópica dos linfonodos regionais. Foi também analisada a presença de lesões tumorais não ma- 
márias. A condição corporal foi avaliada, segundo os critérios de Laflamme (1997), pela visualização do estado geral do animal durante o exame físico. A classificação do porte físico obedeceu a normas do manual da Confederação do Brasil Kennel Clube (1987).

\section{Estadiamento clínico}

Foi realizado o estadiamento clínico, com base no tamanho do tumor $(\mathrm{T})$, no envolvimento neoplásico de linfonodos regionais $(\mathrm{N})$ e na presença de metástases a distância (M), de acordo com o sistema TNM (modificado de Owen, 1980). Os tumores foram mensurados em pelo menos duas dimensões. Quando o animal apresentou mais de um nódulo, cada um deles foi medido separadamente, sendo considerada para a realização do estadiamento clínico a maior medida do tumor invasivo, conforme critérios adotados pelo sistema TNM. A avaliação macroscópica dos linfonodos inguinais e axilares foi realizada por palpação e, durante a mastectomia, os linfonodos inguinais foram removidos juntamente com a mama inguinal, diferentemente dos linfonodos axilares, que foram retirados apenas quando reativos e palpáveis. A presença ou não de envolvimento neoplásico foi determinada pelo exame histopatológico, após a exérese cirúrgica da mama envolvida, sendo a pesquisa de metástase a distância realizada a partir da avaliação radiográfica simples do tórax nas incidências ventrodorsal e latero-lateral direita e esquerda.

\section{Classificação histopatológica}

O tratamento de eleição, realizado em todos os casos, foi a mastectomia unilateral da cadeia acometida e, nos casos de acometimento bilateral, foi removida a cadeia cujo tumor apresentava maior tamanho. Após a exérese cirúrgica, a cadeia mamária foi imediatamente encaminhada ao Laboratório de Anatomia Patológica para avaliação anátomo-histopatológica. Foram coletados fragmentos da(s) mama(s) afetada(s), incluindo pele e tecido subcutâneo, além dos linfonodos regionais. Na presença de tumores mamários múltiplos em um mesmo animal, a coleta dos fragmentos foi realizada separadamente e os frascos identificados com a glândula afetada. Os animais que vieram a óbito foram submetidos à necropsia, quando autorizada pelos proprietários, sendo coletados fragmentos das mamas acometidas, linfonodos e órgãos alvos de metástase. Os fragmentos coletados foram fixados em formol neutro a $10 \%$, tamponado com fosfato e processados pela técnica rotineira de inclusão em parafina. As secções histológicas, de $4 \mu \mathrm{m}$, foram coradas pelas técnicas da Hematoxilina-Eosina (Luna, 1968).

A classificação histopatológica foi realizada com base nas normas da Organização Mundial de Saúde para tumores e displasias da glândula mamária de cães e gatos
(Misdorp et al., 1999). As lâminas foram analisadas simultaneamente por dois patologistas veterinários (LABAP/ UFBA e LPC/UFMG), buscando-se maior acuidade no diagnóstico histopatológico.

\section{Geoprocessamento}

Os dados socioeconômicos dos proprietários, representados por escolaridade e renda, foram obtidos do censo realizado no ano 2000 pelo Instituto Brasileiro de Geografia e Estatística (IBGE, 2000). A área estudada foi o Município de Salvador, subdividido setorialmente em 93 zonas de informação (ZIs). Para escolaridade, foi considerada a percentagem de alfabetizados, divididos em três grupos: baixo (ZIs < $80 \%$ da população alfabetizada); médio (ZIs 80 - 90\% da população alfabetizada); alto (ZIs > 90\% da população alfabetizada). Para o item renda, foi considerado o ganho familiar de até um salário mínimo e as ZIs foram divididas em baixa (> 10\% da população com essa renda); média-baixa (7 - 10\% da população com essa renda); média-alta (3 - 7\% da população com essa renda); e alta ( $<3 \%$ da população com essa renda).

O georreferenciamento foi realizado no Laboratório de Monitoramento de Doenças pelo Sistema de Informação Geográfica (LAMDOSIG/UFBA), sendo montado sobre a base cartográfica digitalizada e georreferenciada, na qual foram plotados os endereços presentes nas fichas clínicas específicas para neoplasia mamária, junto com dados censitários, por meio do software ArcView 9.1. (Figura 1).

\section{Análise estatística}

As análises estatísticas foram realizadas com auxílio do software SPSS versão 13.0 (Statical Package for the Social Sciences). Foi realizada a análise univariada a partir do cálculo da frequência dos dados. Esses foram submetidos à análise de variância (ANOVA), seguida pelo teste LSD e correlação de Pearson (Sampaio, 2002). Os valores foram considerados estatisticamente significativos quando $\mathrm{p}<0,05$.

\section{RESULTADOS E DISCUSSÃO}

Foram atendidos 2204 animais, no período de janeiro de 2006 a abril de 2008; 1359 eram fêmeas, sendo 336 $(24,7 \%)$ portadoras de tumor de mama e, destas, apenas $132(39,28 \%)$ retornaram para realização da mastectomia. A idade das cadelas estudadas variou entre três e 18 anos (Figura 2), sendo de 10,17 anos a média de acometimento, corroborando a maioria dos estudos, que indica maior susceptibilidade a neoplasias mamárias em cadelas entre nove e 11 anos, com rara ocorrência em idades inferiores a dois anos (Benjamin et al., 1999; Lana et al., 2001; Cassali, 2002; Oliveira et al., 2003; Pires et al., 2003; De Nardi et al., 2007; Furian et al., 2007; Ribeiro et al., 2009). 
Constatou-se, ainda, que os tumores de mama acometem animais de todos os tamanhos e, neste estudo, houve um maior envolvimento de cães da raça Poodle, 34,2\% (115/336), seguida por cães sem raça definida (SRD), 28,6\% (96/336) e por Pastor Alemão 9,0\% (20/336). As outras raças somaram 31,3\% (105/336). Segundo Misdorp (2002), a raça Poodle apresenta predisposição genética para o desenvolvimento de neoplasias mamárias. Entretanto, Peleteiro (1994), Cavalcanti e Cassali (2006) afirmam não existir predisposição racial, ainda que os tumores de mama sejam mais frequentemente diagnosticados em determinadas raças. Por isso, acredita-se que esta elevada frequência seja determinada pelo número elevado de cães da raça Poodle e racialmente indefinidos, em Salvador.

$\mathrm{Na}$ anamnese, foi constatado que apenas 31,3\% (105/ 336) dos proprietários alimentavam as cadelas exclusivamente com ração industrial; a maioria - 68,8\% (231/336) relatou inserir comida caseira na dieta de seus animais. Apenas 8\% (27/336) das cadelas eram magras, 65,2\% (219/ 336) apresentavam peso ideal e 26,8\% (90/336) eram obesas. Os resultados demonstraram uma relação direta entre tipo de alimentação e condição corporal do animal $(\mathrm{p}=$ 0,015 ), uma vez que os animais obesos eram, na sua maioria, alimentados com acréscimo de comida caseira em sua $\operatorname{dieta}(80 \%)$.
Foi observada também correlação positiva entre a raça e tipo de alimentação $(\mathrm{p}=0,002)$ e raça e condição corporal $(\mathrm{p}=0,042)$. Cadelas sem raça definida apresentaram maior frequência de alimentação com acréscimo de comida caseira $(83,3 \%)$ e obesidade $(31,3 \%)$, enquanto as da raça Pastor Alemão apresentaram frequência de 50 e $15 \%$, respectivamente. Jericó \& Scheffer (2002) demonstraram em seu estudo que a maioria dos cães obesos recebia petiscos ou comida caseira misturada à sua alimentação. As cadelas SRD tiveram maior acréscimo de comida caseira na ração, provavelmente pelo fato de os proprietários apresentarem nível socioeconômico mais baixo.

Greenwald et al. (2001) listam a dieta como possível responsável pela carcinogênese mamária, sendo considerada uma das principais rotas de exposição do animal a carcinógenos químicos. Sabe-se, ainda, que mulheres obesas apresentam elevadas concentrações de estrógeno proveniente da transformação, no tecido adiposo, da androstenediona em estrona e, posteriormente, em estrógeno, hormônio sabidamente envolvido na carcinogênese mamária (Yoo et al., 2001; Silva et al., 2004).

Em cães e gatos, a progesterona estimula a síntese de hormônio do crescimento na glândula mamária, com proliferação lóbulo-alveolar e consequente hiperplasia de elementos mioepiteliais e secretórios, induzindo a formação

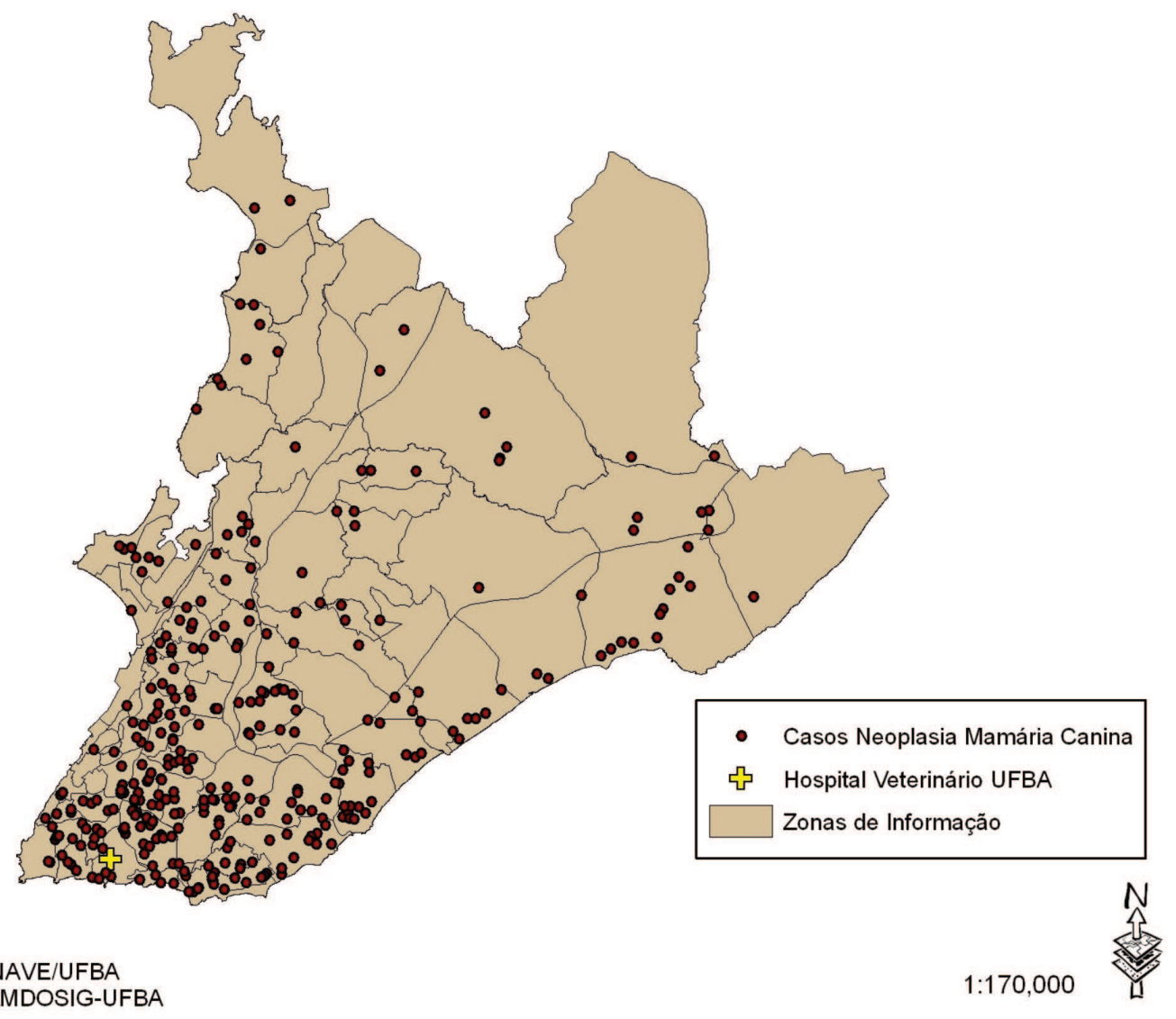

Fonte: IBGE; NAVE/UFBA
Adaptação: LAMDOSIG-UFBA

$1: 170,000$

Figura 1. Área de Estudo - Zonas de Informações do Município de Salvador, Bahia. Identificados, no mapa, os endereços das cadelas acometidas por neoplasia mamária e o Hospital Veterinário da UFBA.

Rev. Ceres, Viçosa, v. 59, n.4, p. 427-433, jul/ago, 2012 


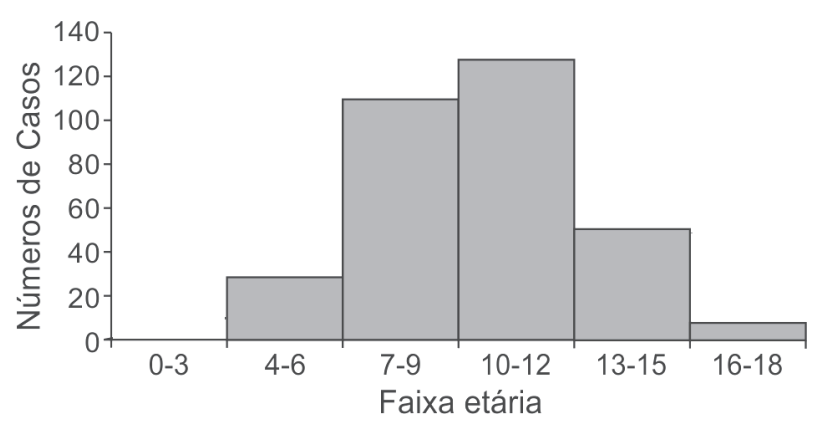

Figura 2. Faixa etária das cadelas, acometidas por neoplasia mamária, atendidas no Hospital Veterinário da UFBA, no período de janeiro de 2006 a abril de 2008.

de nódulos benignos em animais jovens (Lana et al., 2001; Misdorp 2002, Silva et al., 2004). Com base no histórico reprodutivo dos animais pesquisados, constatou-se que 30,4\% (102/336) já apresentaram pseudociese, sendo esta a alteração reprodutiva mais frequentemente observada. Sabe-se, ainda, que a progesterona, apesar de não atuar diretamente na carcinogênese mamária, pode aumentar o número de receptores de estrógeno (Silva et al., 2004). Tais achados reforçam o papel dos hormônios sexuais na iniciação ou promoção da carcinogênese mamária.

Em relação ao ciclo reprodutivo, os animais castrados representavam 12,5\% (42/336) e 44,3\% (149/336) ainda eram nulíparas. Em apenas 11,6\% (39/336) do total de casos, os proprietários admitiram o uso de contraceptivos; contudo, não foi informada com exatidão a sua frequência e dosagem. Apesar de não haver diferença estatística representativa, foi observado, descritivamente, maior utilização de progestágenos, nas ZIs cujos índices de alfabetização e renda foram considerados baixos, com 18,4\% (9/ 49) e $21,7 \%$ (13/60), respectivamente. Nas ZIs cujos índices foram considerados altos, apenas $9,1 \%$ (alfabetização e renda, demonstrados por 8/88 e 6/66, respectivamente) admitiram a utilização desses progestágenos.

A maioria das cadelas, 64,9\% (218/336) apresentou acometimento bilateral da cadeia mamária, sendo, em sua maioria, tumores multicêntricos, 80,1\% (269/336). Os nódulos localizaram-se mais frequentemente nas mamas inguinais, 38,8\% (278/716), seguidos pelos localizados nas mamas abdominais (craniais e caudais), 37,4\% (268/716). Em 73,8\% (248/336) dos animais, os tumores apresentaram integridade cutânea. Misdorp (2002) afirma que as mamas inguinais e abdominais caudais são mais acometidas, por causa da maior quantidade de parênquima mamário, sofrendo, assim, maior alteração proliferativa em resposta aos hormônios.

Diversos autores consideram o tamanho tumoral e o estadiamento clínico os parâmetros prognósticos mais importantes (Peleteiro, 1994; Misdorp et al. 1999; Cavalcanti \& Cassali, 2006; Estrela-Lima et al., 2010; Cassali et al., 2011). Tumores menores que três centíme- tros sem invasão linfática apresentam melhor prognóstico, quando comparados com aqueles maiores ou que já apresentam metástase para linfonodos (Queiroga \& Lopes, 2002; Ferreira et al., 2009). Neste trabalho, somente 20,8\% (70/336) dos cães apresentavam processo neoplásico em fase inicial, com nódulos de até três centímetros.

Os exames radiográficos de tórax foram realizados em todos os 132 animais submetidos à exérese cirúrgica da cadeia mamária. Constatou-se correlação positiva $(\mathrm{p}=0,006)$ entre o tamanho do tumor e a presença de metástase pulmonar. Em 20 casos (15,2\%), foi possível identificar metástase pulmonar e, destes, em 15 cadelas (75\%) o tumor era maior do que cinco centímetros. $\mathrm{Na}$ classificação TNM, as neoplasias com estádio I e II representaram apenas $27,7 \%$ (31/112) de todos os tumores analisados. O estádio III apresentou a maioria dos casos, 36,6\% (41/112), seguido pelos estádios IV e V, com 19,6 e $17,9 \%$, respectivamente (22 e 20/112). O estadiamento clínico apresentou, também, correlação positiva com a média de idade dos animais, indicando que cadelas com estádio I e II apresentavam média de idade inferior $(8,48$ anos) àqueles animais com estádio III, IV e V $(10,5$ anos $)(p=0,002)$. A maioria dos tumores apresentava integridade cutânea; entretanto cabe ressaltar que, neste estudo, $100 \%$ (33/132) dos tumores que apresentavam ulcerações eram malignos. Sabe-se que os tumores de crescimento rápido, aderentes, com grandes áreas de necrose, ulcerados ou com acometimento de planos cutâneos adjacentes estão associados a pior prognóstico e menor tempo de sobrevida (Peleteiro, 1994; Cassali, 2002; Estrela-Lima, 2010).

A classificação histológica dos tumores mamários tem valor estabelecido pela sua importância em predizer o comportamento biológico do tumor (Bostock, 1986); entretanto, cabe ressaltar que a existência de várias classificações histológicas para lesões neoplásicas da glândula mamária de cadelas tem dificultado a comparação dos resultados entre os pesquisadores (Morrisson, 1998; Benjamin et al., 1999; Oliveira et al., 2003; Cassali et al., 2011).

$\mathrm{Na}$ análise histopatológica, os tumores benignos representaram apenas $9,1 \%(12 / 132)$ de todos os animais submetidos à cirurgia. Destes, apenas um foi classificado como adenoma (8,3\%), sendo o restante representado pelo tumor misto benigno $(91,7 \%$ - 11/12). Cadelas que apresentaram diagnóstico benigno tinham média de idade de 7,5 anos, enquanto a média de idade daquelas que apresentaram diagnóstico maligno foi de 10,09 anos ( $\mathrm{p}<0,001$ ). Esses dados corroboram os que indicam o surgimento das neoplasias mamárias benignas em cadelas de idade mais jovem (Peleteiro, 1994; Cassali, 2000).

Neste estudo, os tumores malignos representaram $90,9 \%(120 / 132)$ do total pesquisado e o carcinoma em 
tumor misto (CTMB) foi o tipo histológico predominante $(56,7 \%)$, sendo este o subtipo de carcinoma mais comumente descrito por outros autores (Cassali, 2000; Cavalcanti \& Cassali, 2006; Ribeiro et al., 2009). Foi observado que 86,8\% (59/68) dos CTMB possuíam caráter infiltrativo, enquanto apenas $13,2 \%$ (9/68) apresentavamse "in situ". Este tipo tumoral deriva da transformação maligna de componentes epiteliais do tumor misto benigno (Moulton \& Rosenblat, 1970). A classificação histológica deste subtipo deve-se basear no pleomorfismo celular e na ruptura da membrana basal, com invasão para o estroma (Bertagnolli et al., 2009).

Os outros carcinomas corresponderam a 36,6\% (44/ $120)$, sendo dez sólidos $(22,7 \%)$, nove papilares e nove tubulares (20,5\% cada), sete túbulo-papilares $(15,9 \%)$ e apenas dois anaplásicos (4,5\%). Os carcinomas inflamatórios foram diagnosticados em 15,9\% dos casos (7/44) e, os carcinossarcomas, em 6,7\% (8/120). É importante ressaltar que todas as cadelas portadoras destes dois tipos tumorais apresentaram metástase a distância e vieram a óbito, sendo o tempo médio de sobrevida igual a 60 dias, indicando o alto grau de malignidade desses dois tipos tumorais (Cavalcanti \& Cassali, 2006). O período de sobrevida global foi definido como o tempo entre a exérese cirúrgica da neoplasia, ou surgimento nos casos de CIM, e a data de óbito do animal.

Com relação aos fatores socioeconômicos, o nível de escolaridade e a renda influenciaram o tempo de procura pelo serviço Médico Veterinário, indicado pela correlação positiva entre esses fatores e as variáveis tamanho, ulceração, diagnóstico e estadiamento clínico. Dos 336 animais atendidos, 36,7\% (18/49) pertenciam a ZIs de baixo nível de alfabetização e apresentavam tumor ulcerado, enquanto, entre aqueles pertencentes às ZIs de alto nível de alfabetização, apenas 15,9\% (14/88) apresentavam ulceração ( $\mathrm{p}=0.758$ ). Dos animais que apresentavam tumores ulcerados, 64,8\% (57/88) eram residentes das ZIs de renda média-baixa e baixa. Esses resultados indicam que ainda há necessidade de fornecer informação aos proprietários sobre prevenção, diagnóstico precoce e tratamento das neoplasias mamárias, principalmente nos locais onde há menor índice de instrução e renda.

\section{CONCLUSÃO}

Os dados apresentados demonstram a correlação entre os achados clinico-patológicos dos tumores de mama em cadelas e os fatores socioeconômicos da população estudada, que refletem a falta de recursos e,ou, desconhecimento a respeito da importância da assistência Médica Veterinária e da possível progressão do processo neoplásico. Além disso, o diagnóstico tardio implicou tumores maiores que cinco centímetros, estádios mais avan- çados, caráter infiltrativo e, consequentemente, piores prognósticos. Por isso, é urgente educar a população quanto à prevenção e detecção da neoplasia mamária nas cadelas, buscando o diagnóstico precoce e a terapêutica adequada.

\section{REFERÊNCIAS}

Allred DC, Harvey JM \& Berardo M (1998) Prognostic and predictive factors in breast cancer by immunohistochemical analysis. Modern Pathology, 11:155-168.

Andrade FHE, Figueiro AFC, Bersano PRO, Bissacot DZ \& Rocha NS (2010) Malignant mammary tumor in female dogs: environmental contaminants. Diagnostic pathology, 45:0105

Benjamin SA, Lee AC \& Saunders WJ (1999) Classifiction and behavior of canine mammary epithelial neoplasms based on life-span observations in Beagles. Veterinary Pathology, 36:423436.

Bertagnolli AC, Cassali GD, Genelhu MCLS, Costa FA, Oliveira JF \& Gonçalves PB (2009) Immunohistochemical expression of p63 and DeltaNp63 in mixed tumors of canine mammary glands and its relation with p53 expression. Veterinary Pathology, 46:407-415.

Bostock DE (1986) Neoplasms of the skin and subcutaneous tissues in dogs and cats. The British Veterinary Journal, 142:01-19.

Brodey RS, Goldschmidt MH \& Roszel JR (1983) Canine mammary gland neoplasms. Journal of the American Animal Hospital Association, 19:61-90.

Cassali GD (2000) Estudo morfológico, imunohistoquímico e citométrico de tumores mamários da cadela - aspectos comparativos com neoplasias da mama humana. Tese de Doutorado. Universidade Federal de Minas Gerais, Belo Horizonte. 80p.

Cassali GD (2002) Patologias da Glândula Mamária. In: Nascimento EF \& Santos RL (Eds.) Patologia da Reprodução dos Animais Domésticos. Rio de Janeiro, Guanabara Koogan. p.119133.

Cassali GD, Lavalle GE, De Nardi AB, Ferreira E, Bertagnolli AC, Estrela-Lima A, Alessi AC, Daleck CR, Salgado BS, Ghever C, Sobral RA, Amorim RL, Gamba CO, Damasceno KA, Aules PA, Magalhães GM, Silva JO, Raposo JB, Ferreira AMR, Oliveira LO, Malm C, Zuccar DAPC, Ribeiro LGR, Campos LC, Souza CM, Leite JL, Soares LMC, Cavalcanti MF, Fonteles ZG, Schuch ID, Paniago J, Oliveira TS, Terra EM, Castanheira TLL, Felix AOC, Carvalho GD, Guim TN, Garrido E, Fernandes SC, Maia FCL, Dagli MLZ, Rocha NS, Fukumasu H, Grandi F, Machado JP, Silva SMMS, Bezerril JE, Frehse MS \& Campos CB. (2011). Consensus for the diagnosis, prognosis and treatment of canine mammary tumors. Brazilian journal of veterinary pathology, $4: 153-180$.

Cavalcanti MF \& Cassali GD (2006) Fatores prognósticos no diagnóstico clínico e histopatológico dos tumores de mama em cadelas - revisão. Revista Clínica Veterinária, 61:56-63.

Confederação do Brasil Kennel Clube (CBKC) (1987) Manual de estrutura e dinâmica do cão. p.113.

Dagli MLZ (2008) The search for suitable prognostic markers for canine mammary tumors: A promising outlook. The Veterinay Journal, 177:03-05.

De Nardi AB (2007) Correlação da ciclooxigenase-2 com Ki-67, P53 e caspase-3 nas neoplasias de mama em cadelas. Tese de Doutorado. Universidade Estadual Paulista, Jaboticabal. 92p. 
Estrela-Lima A, Araújo MSS, Costa-Neto JM, Teixeira-Carvalho A, Barrouin-Melo SM, Cardoso SV, Martins-Filho AO, Serakides R \& Cassali GD (2010) Immunophenotypic features of tumor infiltrating lymphocytes from mammary carcinomas in female dogs associated with prognostic factors and survival rates. BMC Cancer, 10:256-270.

Ferreira E, Bertagnolli AC, Cavalcanti MF, Schmitt FC \& Cassali GD (2009) The relationship between tumour size and expression of prognostic markers in benign and malignant canine mammary tumours. Veterinary and Comparative Oncology, 7:01-06.

Ferreira E, Bregunci GC, Schmitt FC \& Cassali GD (2003) Protocol for the anatomopathological examination of canine mammary tumors. Arquivo Brasileiro Medicina Veterinaria Zootecnia, 55:105-109.

Furian M, Sandei CFCS, Rocha EJN \& Lot RFE (2007) Estudos retrospectivo dos tumores mamários em caninos e felinos atendidos no Hospital Veterinário da FAMED entre 2003 a 2007. Revista Científica Eletrônica de Medicina Veterinária, 8.

Greenwald P, Clifford CK \& Milner JA (2001) Diet and cancer prevention. European Journal of Cancer, 37:948-965.

Henderson BE \& Feigelson HS (2000) Hormonal carcinogenesis. Carcinogenesis, 21:427-433.

Humphrey LL, Helfand M, Chan BKS \& Woolf SH (2002) Breast Cancer Screening: A Summary of the Evidence for the U.S Preventive Services Task Force. Annals of Internal Medicine, 137:347-360

Instituto Brasileiro de Geografia e Estatística - IBGE (2000) Censo Demográfico 2000 Resultados do universo. Disponível em: http://www.ibge.gov.br/home/estatistica.php. Acessado em: 20 de outubro 2011

Jericó MM \& Scheffer KC (2002) Aspectos epidemiológicos dos cães obesos na cidade de São Paulo. Revista Clínica Veterinária, $7: 25-29$.

Laflamme DP (1997) Development and validation of a body condition score system for dogs. Canine Practice, 22:10-15.

Lana SE, Rutteman GR, Withrow SJ (2001) Tumors of the Mammary Gland. In: Withrow SJ, Macewen EG (Eds.) Small Animal Clinical Oncology. $4^{\mathrm{a} e d . ~ S t . ~ L o u i s, ~ S a u n d e r s ~ E l s e v i e r . ~}$ p.619-636.

Luna LG (1968) Manual of histologic staining methods of the Armed Forces Institute of Pathology. New York, McGraw Hill. $258 \mathrm{p}$.

Misdorp W (2002) Tumors of the mammary gland. In: Meuten DJ (Ed.) Tumors in Domestic Animals. Iowa State, University of California. p. 575-606.

Misdorp W, Else RW \& Hellmén E (1999) Definitions and explanatory notes. In: ___ (eds.) Who histological classification of mammary tumors of the dog and cat. Washington, Armed Forces Institute of Pathology. p.18-27.
Morrisson WB (1998) Cancers in dogs and cats. Medical and surgical management. Philadelphia, Willians e Wilkins. 785p.

Moulton JE \& Rosenblat LS (1970) Canine mammary tumors. Veterinary Pathology, 7:289-320.

Oliveira LO, Oliveira RT, Loretti AP, Rodrigues R \& Driemeier D (2003) Aspectos epidemiológicos da neoplasia mamária canina. Acta Scientiae Veterinariae, 31:105-110.

Owen LN (1980) TNM Classification of tumors in Domestic Animals. Geneva, World Health Organization, 53p.

Peleteiro MC (1994) Tumores mamários na cadela e na gata. Revista Portuguesa de Ciências Veterinárias, 89:10-29.

Pires MA, Travassos FS \& Pires I (2003) Neoplasias em canídeos - Um estudo descritivo de seis anos. Revista Portuguesa de Ciências Veterinárias, 98:111-118.

Queiroga F \& Lopes C (2002) Tumores mamários caninos - Novas perspectivas. In: Congresso de ciências veterinárias, Oeiras. Proceedings of the Veterinary Sciences Congress, SPCV. p.183190.

Ribeiro LGR, Damasceno K, Costa Neto JM, D'Assis MJMH, Silva NS, Aguiar PHP, Cassali GD \& Estrela-Lima A (2009) Expressão da Cox-2 nos carcinomas mamários de cadela. Veterinária em Foco, 6:134-139.

Rivera P \& von Euler H (2011). Molecular biological aspects on canine and human mammary tumors. Veterinary Pathology, 48:132- 46

Sampaio IBM (2002) Estatística aplicada à experimentação animal. $2^{a}$ ed. Belo Horizonte, Fundação de Estudo e Pesquisa em Medicina Veterinária e Zootecnia (FEPMVZ). 265p.

Schneider R, Dorn CR \& Taylor DO (1969) Factors influencing canine mammary cancer development and post surgical survival. Journal of the National Cancer Institute, 43:1249-1261.

Silva AE, Serakides R \& Cassali GD (2004) Carcinogênese Hormonal e neoplasias hormônio-dependentes. Revista Ciência Rural, 34:625-633.

Uva P, Aurisicchio L, Watters J, Loboda A, Kulkarni A, Castle J, Palombo F, Viti V, Mesiti G, Zappulli V, Marconato L, Abramo F, Ciliberto G, Lahm A, La Monica N \& De Rinaldis E (2009) Comparative expression pathway analysis of human and canine mammary tumors. BMC Genomics, 135:01-20.

Yoo K, Tajima K, Park S, Kang D, Kim S, Hirose K, Takeuchi TC \& Miura S (2001) Postmenopausal obesity as a breast cancer risk factor according to estrogen and progesterone receptor status. Cancer Letters, 167:57-63. 